\title{
Cell Signaling in Hepatocellular Carcinoma
}

\author{
Mahmoud Balbaa $a^{\#}$ Yasmin R. Shahina, Nihal M. Elguindy
}

${ }^{* 1}$ Department of Biochemistry, Faculty of Science, Alexandria University, Alexandria, Egypt

\#The corresponding author (Fax: +20 3 3911794; E-mail: mahmoud.balbaa@alexu.edu.eg, http://orcid.org/0000-0002-0876-6604).

\begin{abstract}
Cell signaling pathways are important for cellular communications and control different cellular processes of growth, differentiation, and apoptosis. There are many ameliorations or magnifications of these pathways in cancer. Hepatocellular carcinoma (HCC) is one of the cancer diseases in which many signals are stimulated or inhibited. Moreover, the rate of the signaling pathway is linked to the production of the reactive oxygen species (ROS) and the antioxidant mechanism. Ras/Raf/MEK is one of the important pathways that was intensively studied in HCC and affected by ROS.
\end{abstract}

KEYWORDS: Hepatocellular Carcinoma, growth factors, cell signaling, oxidative stress, antioxidants.

\section{INTRODUCTION}

There are many types of cell signaling including endocrine signaling, paracrine signaling, and autocrine signaling, whereas the different types are determined according to the type of the primary signal ${ }^{[1]}$. The signaling effect in each type is initiated by the recognition of the primary signal to a cell surface or an intracellular receptor ${ }^{[1,2]}$. The transduction of cellular signaling is transduced by many proteins and enzymes such as $\mathrm{G}$ proteins, protein kinases, phospholipases especially phospholipase $\mathrm{C}$, and transcription factors ${ }^{[1,2,3]}$. The events of cell signaling pathway include the specific interaction of the primary signal to the receptor, the transmission of the signal by receptors into the cell, passing on of the message to a series of signaling components as a signaling cascade, arrival of the message at the destination, and finally a cellular response. In many pathways, signal divergence toward several target molecules occurs after the amplification of signals ${ }^{[1,2]}$. The amplification occurs by many secondary signals such as 3',5'-cyclic adenosine monophosphate (cAMP), 3',5'-cyclic guanosine monophosphate (cGMP), calmodulin, and lipidderived messengers such as inositol 1,4,5-trisphosphate (IP3), Diacylglycerol (DAG), phosphatidic acid (PA), and sphingosine phosphate ${ }^{[4]}$.

Liver cancer is the sixth most common type of cancer in the world, with approximately 840,000 new cases each year. It is one of the most common causes of cancer-related mortality with the cancer of the lung and stomach ${ }^{[5]}$. Hepatocellular carcinoma (HCC) is the most common type of primary cancer ${ }^{[6]}$. Hepatitis $\mathrm{B}$ and $\mathrm{C}$ virus infection is the greatest risk factor associated with the development of HCC. Hepatitis infection increases the risk of developing HCC by 20 fold and is the major etiological factor in most HCC cases [7]. Excessive alcohol consumption, nonalcoholic steatohepatitis, exposure to environmental toxins such as aflatoxin B, hemochromatosis, cirrhosis, diabetes, and obesity are the other main risk factors $[8,9,10]$.

Antioxidants exert a defense mechanism against free radical damage and are important in maintaining optimum health and wellbeing. For the oxidative stress, oxygen is a highly reactive atom that can become part of potentially damaging molecules commonly called "free radicals" or reactive oxygen species (ROS). ROS can attack the healthy cells of the body, causing them to lose their structure and function. They have been involved in the pathogenesis of at least 50 diseases ${ }^{[11]}$. This damage can become cumulative at a limited availability of antioxidants, which are capable of stabilizing or deactivating the free radicals before they attack cells ${ }^{[12]}$. The antioxidant protection system involves a variety of endogenous and exogenous components, to neutralize free radicals ${ }^{[13]}$. One type of these components is nutrient-derived antioxidants like ascorbic acid (vitamin $\mathrm{C}$ ), tocopherols and tocotrienols (vitamin E), carotenoids, and other low molecular weight compounds such as glutathione and lipoic acid. Other important components are the antioxidant enzymes such as superoxide dismutase, glutathione peroxidase, and glutathione reductase, which catalyze free radical quenching reactions ${ }^{\text {[14] }}$ In addition, the metal-binding proteins, such as ferritin, lactoferrin, albumin, and ceruloplasmin that sequester free iron and copper ions that are capable of catalyzing oxidative reactions ${ }^{[15]}$.

\section{Growth factor signaling}

Growth factors - stimulated pathways are one of the important signaling processes. They are small or medium 
molecular weight proteins, bind to specific cell surface receptors, and considered as hormone-like modulators of cell proliferation and differentiation in different cell types. Most of their receptors exhibit protein-tyrosine kinase activity located in their cytoplasmic domain of the subunit structure ${ }^{[16]}$. The regulation of cell proliferation is affected by the cellular and molecular actions of growth factors ${ }^{[17]}$. The action of growth factors is different from hormones by having other biological activities in addition to the stimulation of cell proliferation

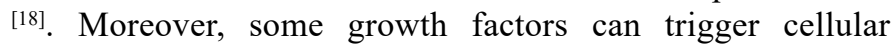
differentiation, enhance cell survival, initiate cell migration, and stimulate the secretion of tissue-specific hormones from differentiated cell types ${ }^{[16]}$. Examples of growth factors include the epidermal growth factor (EGF), platelet-derived growth factor (PDGF), insulin-like growth factor (IGF), transforming growth factor (TGF), nerve growth factor (NGF) and fibroblast growth factor (FGF). Hepatocyte growth factor (HGF) is a growth factor produced in the mesenchymal cell ${ }^{[19]}$, whereas vascular endothelial growth factor (VEGF) is an endothelial cell mitogen that is highly permeable ${ }^{[20]}$. Some cytokines are considered as growth factors such as the hemopoietic cytokines, which form a family of glycosylated extracellular proteins and regulate the production and functional activity of hemopoietic cells [21]. In addition, T cell growth factor (TCGF) or interleukin 2 (IL-2) is required for the proliferation of mature T cells ${ }^{[22,23]}$.

\section{EGF signaling in HCC}

Patients with hepatocellular carcinoma have significantly higher serum levels of EGF and TGF- $\beta 1$ in comparison to their level in patients with chronic hepatitis $\mathrm{C}$ infection and control subjects. The levels of phosphorylated EGFR in hepatocellular carcinoma and chronic hepatitis $\mathrm{C}$ patients show a highly significant difference between patients ${ }^{[24]}$. Therefore, it was suggested that EGF and its phosphorylated form could be used as sensitive biomarkers for the diagnosis and prognosis of these diseases.

Ras/Raf/MEK is an extracellular signal-regulated kinase (ERK) signaling pathway that is implicated in HCC development ${ }^{[25]}$. By transmitting the signals received by the growth factor receptor, Ras/Raf/MEK/ERK cascade reaction activates transcription factors and regulates gene expression. There are different mechanisms for the activation of Ras/Raf/ MEK/ERK cascade in tumor cells. They include overexpression of wild or mutant receptors, such as EGFR, chromosome translocation, such as BCR-ABL, and cytokine mutations such as Flt-3, Fms, and Kit. This signal transduction pathway plays a variety of roles in cell cycle regulation, apoptosis, and cell differentiation [26]. Briefly, activation of this pathway involves activation of Ras that recruits and activates the protein kinase Raf (serine/threonine-protein kinase), which in turn promotes MEK1/2 (MAPK/ERK kinase) dual-specificity protein kinase and hence the activation of ERK1/2. Active ERKs can phosphorylate numerous cytoplasmic and nuclear targets, including kinases, phosphatases, transcription factors and cytoskeletal proteins leading to different gene expression [27] (Figure 1).

Ras/Raf/MEK/ERK signaling pathway plays a vital role in the pathogenesis of HCC. Increased expression of Ras/Raf/ MEK/ERK kinase in HCC tissues and cells has been observed

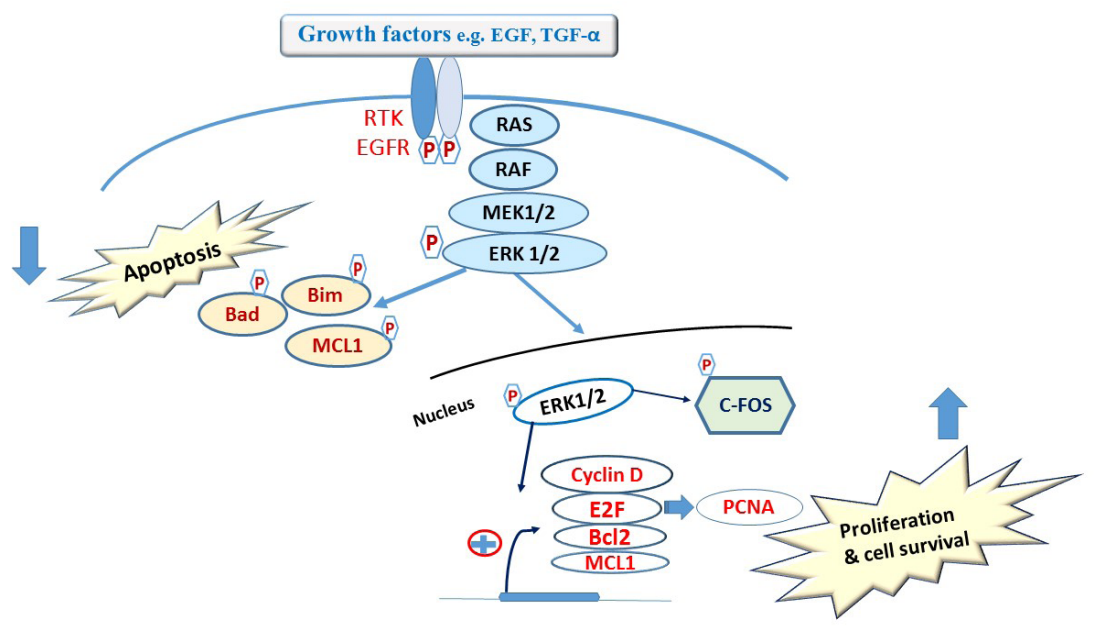

Figure 1: Activation of RAS/RAF/MEK/ERK signaling pathway in HCC. Overexpression of growth factors such as EGF, or TGF- $\alpha$ leads to activation EGFR as RTK, which in turn activates RAS/RAF/MEK/ERK signaling pathway. Activated ERKs can translocate into the nucleus to phosphorylate c-fos that in turn increases transcription of genes involved in cell cycle progression in hepatocytes such as cyclin D1. Cyclin $D 1$ promotes activation of $E 2 F$, which act as transcription factors to activate many target genes to drive progression into, and through, $S$ phase of the cell cycle such as PCNA. ERKs activation also promotes the transcription of cell survival genes such as Bcl2 and MCL1 as well as inhibits apoptosis by the direct phosphorylation of pro-apoptotic molecules Bim and Bad. Moreover, ERK1/2 stabilizes MCL1 by phosphorylation promoting cell survival. 
in several studies, whereas the Ras gene was mutated in $30 \%$ of HCC cases ${ }^{[28]}$. Moreover, Raf kinase was overexpressed in most HCC cases ${ }^{[29]}$. Furthermore, the overexpression of many upstream growth factors in HCC such as EGF, VEGF, PDGF- $\beta$, and TGF- $\alpha$ was combined with RTK leading to activation of Ras/Raf/MEK/ERK pathway ${ }^{[30]}$. Increased expression and activation of ERK is observed in experimental models of different stages of HCC from the early stages to malignant progression [31]. Yoshida et al found that phosphorylated levels of ERK in $53 \%$ of HCC tissues were higher than those in para-carcinoma tissues ${ }^{[32]}$.

Activation of this signaling pathway upon overexpression of many growth factors and their receptors can result in the translocation of activated ERK $1 / 2$ into the nucleus contributing to the activation of different transcription factors. ERK1/2 can phosphorylate and activate preexistent transcription factors such as Elk-1that enhances transcription of growth-related proteins such as c-Fos ${ }^{[33]}$ as well as the stabilization of c-Fos through direct phosphorylation by ERK1/2. Persistent expression of c-Fos allows its association with c-jun to form a transcriptionally active AP- 1 complex that is required for the transcriptional stimulation of cyclin D1 that permits cell cycle progression in hepatocytes ${ }^{[34]}$. It has been found that $44 \%$ of HCC cases showed higher expression of c-Fos protein than that in para-carcinoma tissues, and the c-Fos expression level was positively correlated with ERK protein expression [35]. On the other hand, ERK1/2 affect the expression and activity of several members of the Bcl-2 protein family. Activation of ERK1/2-dependent RSK and MSK1/2 activates CREB to promote transcription of cell survival genes such as Bcl-2 ${ }^{[36]}$. It can also phosphorylate and inactivate the pro-apoptotic molecules such as Bcl-2 interacting mediator of cell death (Bim) and Bcl-2-associated death promoter (Bad). ELK1 activation by ERK1/2 may also augment MCL1 transcription. Moreover, ERK1/2 stabilizes MCL1 by phosphorylation leading to the promotion of cell survival ${ }^{[37]}$.

Tumor metastasis and recurrence are the primary contributors to poor prognosis in patients with HCC. Ras/Raf/ $\mathrm{MEK} / \mathrm{ERK}$ pathway significantly implicates in drug resistance of HCC due to the activation of stem-like cancer cells that contribute to tumor recurrence and poor therapeutic outcome. Epithelial-mesenchymal transition (EMT) has a major role in the important features of cancer cells, tumor metastasis, invasion, and recurrence. The hallmark of EMT is the loss of epithelial surface markers, most notably E-cadherin, and the acquisition of mesenchymal markers including vimentin and $\mathrm{N}$-cadherin ${ }^{[38]}$. The downregulation of E-cadherin during EMT can be mediated by its transcriptional repression through the binding of EMT transcription factors (EMT-TFs) such as SNAIL, SLUG and TWIST to E-boxes present in the E-cadherin promoter. Additionally, EMT-TFs can also cooperate with several enzymes to diminish the E-cadherin expression and regulate EMT at the epigenetic and post-translational level ${ }^{[39]}$.

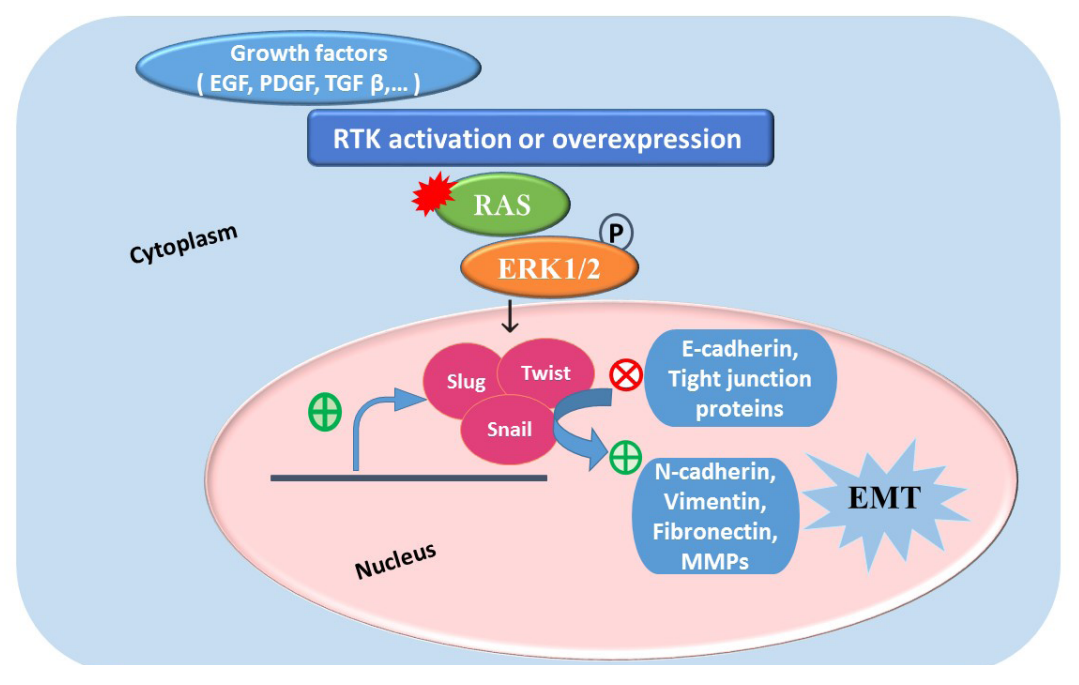

Figure 2: Role of ERK1/2 activation in epithelial-mesenchymal transition in HCC metastasis. Oncogenic activation of RAS due to overexpression or activation of RTKs leads to ERK1/2 phosphorylation. Activated ERk1/2 promotes EMT in HCC through activation of transcription of EMT-TFs such as Slug, Snail, and Twist as well as their stabilization by phosphorylation. While these transcription factors suppress the transcription of E-cadherin and other proteins involved in the cell-cell junction, they activate $N$-cadherin, vimentin transcription as well as induce the expression of matrix metalloproteinases (MMPs) facilitating the epithelial-mesenchymal transition process and tumor invasion. 
RAS-RAF-MEK-ERK MAPK is the major signaling pathway that promotes EMT by increasing the expression of EMT -activating transcription factors and mesenchymal proteins. This results in repressing the expression of epithelial proteins and thereby facilitates cell motility and invasion (Figure 2). Several shreds of evidence support the functions of RAS oncoproteins in the acquisition of tumor cells with EMT features, enhanced metastatic potential, and poor patient survival ${ }^{[40]}$. In addition, ERK can also phosphorylate and modulate the activity of the Twist, Snail, Slug, and Zeb1 transcription factors either directly or indirectly which can regulate cellular proliferation, survival and some can modulate EMT ${ }^{[41-43]}$. Phosphorylation of the transcription factors by ERK1/2, or in some cases by the related MAPK, p38MAPK, prevents their ubiquitination and results in their stabilization and increased activity in the nucleus and ability to promote EMT ${ }^{[44-46]}$. The ERK pathway also induces the expression of matrix metalloproteinases and thereby promotes the degradation of extracellular matrix proteins and consequent tumor invasion ${ }^{[47]}$.

\section{Oxidative stress and MAPK signaling pathways}

Oxidative stress has an important role in the pathogenesis of hepatocellular carcinoma ${ }^{[48]}$. However, the Ras pathway is one of the most significant pathways associated with oxidative stress and cancer ${ }^{[49]}$. Accumulation of ROS has complicated effects on the Ras/Raf/ERK pathway, as they directly enhance the activation of Ras and increases ERK activation. In a study in which MA-10 Leydig tumor cells were used, they found that cAMP analogs enhanced ROS formation and ROS scavenger prevented the increase in $\operatorname{ROS}^{[50]}$. The scavenger also inhibited the increase in ERK1/2 phosphorylation stimulated by cAMP analogs. Increased levels of ROS also promote the Rasinduced phenotype transformation like anchorage-independent growth, tumorigenesis, and morphological transformation ${ }^{[51]}$. Also, previous studies confirmed that H-rasG12V-transgenic mice with hepatic tumors displayed high levels of ROS in hepatocyte ${ }^{[52,53]}$. Consequently, the induction of Ras by oxidative stress has an important role in HCC development ${ }^{[54]}$.

The explanation of how ROS can activate Ras that result in increased phosphorylation of ERK $1 / 2$ is still not completely clear. The levels of GTP-bound Ras (active Ras) are controlled by enhancing the exchange of the bound GDP for Ras- GTP, a process that is enabled by Ras guanine nucleotide exchange factors or by reducing the degradation of the Ras-bound GTP, a process that is facilitated by Ras GTPase-activating proteins [55, 56]. Ras is also changed in the posttranslational process that is vital for its localization at the plasma membrane where activation happens ${ }^{[56]}$. Thus, Ras guanine nucleotide exchange factors, GTPase activating proteins, the localization of Ras at the plasma membrane, and even Ras itself could be ROS targets ${ }^{[57]}$. When Sos can be excluded, the Ras guanine nucleotide exchange factor binds with the EGFR [55, 56], as a potential ROS target because neither Ras activation nor ERK1/2 phosphorylation is affected by ROS scavengers when they are connected with EGF in MA-10 cells ${ }^{[50]}$. This is significant since in some cell types ROS has been shown to stimulate the shedding of EGF-like growth factors leading to EGFR phosphorylation and Ras activation ${ }^{[58]}$.

The mitogen-activated protein kinase (MAPK) signaling pathways control different cellular responses, such as proliferation, differentiation, migration, apoptosis, and autophagy [59]. MAPKs can be triggered by many factors. So, the roles of the MAPK signaling pathways in cancer progression are complicated [60]. There are 4 MAPK family members, which are stimulated by ROS, including Extracellular Regulated Kinases 1/2 (ERK1/2), Jun N-terminal Kinases (JNKs), P38 mitogen-activated protein kinases (p38), and Big MAPK Kinase 1 (BMK1) [61]. Oxidative stress can activate ERK through the RAS-RAF-MEK-ERK kinases cascade [61]. Therefore, many cellular processes and activities are controlled by the activation of ERKs [59, 61]. The JNK and p38 MAPK pathways have the same upstream regulators and also can be activated by oxidative stress [62]. In the p38 MAPK signaling pathway, ROS/RNS activates the p38 MAPK pathway by enhancing the activity of MAPK/ ERK kinase kinase 1 (MEKK1), mixed-lineage protein kinase 3 (MLK3) and apoptosis signal-regulating kinase 1 (ASK1). Then the MAPKKKs sequentially activate MAPK kinase 3 and MAPK kinase 6 (MAP2K3/6) ${ }^{[59,61]}$. The activated p38 MAPK affects multiple transcription factors, such as p38, MEF2, and ATF-2 ${ }^{[63]}$. In the JNK signaling pathway, ROS can induce the activation of ASK1 and MAP3K1, which are MAPKKK. Then the MAPKKK activates MAP2K4/7 that subsequently activates JNKs. MAPK pathway is the BMK1 signaling pathway, which can also be activated by oxidative stress. This pathway is like the mentioned MAPK pathway in which MEKK2/3 activates MAP2K2 and subsequently activates BMK1 ${ }^{[61,64]}$. Experimental evidence suggests that the BMK1 signaling pathway promotes angiogenesis and metastasis in cancer ${ }^{[64]}$. Recently, the antioxidant effect and EGFR/ERK1/2 signaling were used as parameters for the protective mechanism against induced HCC ${ }^{[65]}$.

\section{Conclusion}

There are different signaling pathways in hepatocellular carcinoma and the growth factor signaling is one of the operative biological processes. These processes are related to many mechanisms in which the phosphorylation of the transcription factors and the role of the antioxidants in the cells are important events.

\section{Authors Contribution}

MB: Conception or design of the work, Data collection, Drafting the article, Critical revision, and final approval of the article.

YRS: Critical revision and Final approval of the article.

NME: Critical revision and Final approval of the article. 


\section{Conflicts of Interest}

The authors declare no conflicts of interest.

\section{REFERENCES}

1. Krauss G. Basics of Cell Signaling. In: Biochemistry of Signal Transduction and Regulation, 5th Ed, WILEY-VCH, KGaA, Weinheim, 2014:1-26.

2. Papin JA, Hunter T, Palsson BO, et al. Reconstruction of cellular signalling networks and analysis of their properties. Nat Rev Mol Cell Biol 2005;6:99-111

3. Kadamur G, Ross EM. Mammalian phospholipase C. Annu Rev Physiol $2013 ; 75: 127-54$

4. Pollard T, Earnshaw W, Lippincott-Schwartz J, Johnson G. Signaling Mechanisms. In: Cell Biology, 3rd Ed, Elsevier, Netherlands, 2017: 40786.

5. Ferlay J, Soerjomataram I, Dikshit R, et al. Cancer incidence and mortality worldwide: sources, methods and major patterns in GLOBOCAN 2012. Int J Cancer 2015;136:E359-86.

6. Forner A, Llovet JM, Bruix J (2012). "Hepatocellular carcinoma". The Lancet. 379 (9822): 1245-1255.

7. Anzola M. Hepatocellular carcinoma: role of hepatitis B and hepatitis C viruses proteins in hepatocarcinogenesis. J Viral Hepat 2004;11:383-93.

8. El-Serag HB, Rudolph KL. Hepatocellular carcinoma: epidemiology and molecular carcinogenesis. Gastroenterology 2007;132:2557-76.

9. Hassan MM, Curley SA, Li D, et al. Association of diabetes duration and diabetes treatment with the risk of hepatocellular carcinoma. Cancer 2010;116:1938-46.

10. Whittaker S, Marais R, Zhu AX. The role of signaling pathways in the development and treatment of hepatocellular carcinoma. Oncogene 2010;29:4989-5005.

11. Langseth L. From the editor: Antioxidants and diseases of the brain. Antioxidant Vitamins Newsletter 1993; 4:3.

12. Halliwell B. Free radicals, antioxidants, and human disease: curiosity, cause, or consequence? Lancet 1994;344:721-4.

13. Jacob RA. The integrated antioxidant system. Nutr research 15, 1995;755766

14. Yadav A, Kumari R, Yadav A, et al. Antioxidants and its functions in human body - A Review, 2016

15. de Silva DM, Aust SD. Ferritin and ceruloplasmin in oxidative damage: review and recent findings. Can J Physiol Pharmacol 1993;71:715-20.

16. Balbaa M, Bassiouny K, El-Ashry ES. Growth factors: properties, receptors and Inhibitors. Current Trends in Medicinal Chemistry, 2008;5: $33-44$.

17. Carpenter G, Cohen S. Epidermal growth factor. Annu Rev Biochem 1979;48:193-216.

18. Carpenter G. Epidermal Growth Factor. Handb Exp Pharmacol 1981;57: $89-132$

19. Matsumoto K, Nakamura T. Hepatocyte growth factor: molecular structure, roles in liver regeneration, and other biological functions. Crit Rev Oncog 1992;3:27-54.

20. Cheung CY. Vascular endothelial growth factor: possible role in fetal development and placental function. J Soc Gynecol Investig 1997;4:16977.

21. Nicola NA. Hemopoietic cell growth factors and their receptors. Annu Rev Biochem 1989;58:45-77.

22. Cantrell DA, Smith KA. The interleukin-2 T-cell system: a new cell growth model. Science 1984;224:1312-6.

23. Leonard WJ, Depper JM, Crabtree GR, et al. Molecular cloning and expression of cDNAs for the human interleukin-2 receptor. Nature 1984;311:626-31
24. Shehata F, Abdel-Monem N, Sakr M, et al. Epidermal growth factor, its receptor and transforming growth factor- $\beta 1$ in the diagnosis of $\mathrm{HCV}$ induced hepatocellular carcinoma, 2013.

25. Abou-Alfa GK. Current and novel therapeutics for hepatocellular carcinoma. In: American Society of Clinical Oncology Educational Book. Perry MC (Ed). ASCO, Alexandria, VA, 2004; 192- 197

26. Steelman LS, Pohnert SC, Shelton JG, et al. JAK/STAT, Raf/MEK/ERK, $\mathrm{PI} 3 \mathrm{~K} / \mathrm{Akt}$ and $\mathrm{BCR}-\mathrm{ABL}$ in cell cycle progression and leukemogenesis. Leukemia 2004;18:189-218.

27. Yoon S, Seger R. The extracellular signal-regulated kinase: multiple substrates regulate diverse cellular functions. Growth Factors 2006;24:2144.

28. Downward J. Targeting RAS signalling pathways in cancer therapy. Nat Rev Cancer 2003;3:11-22.

29. Hwang YH, Choi JY, Kim S, et al. Over-expression of c-raf-1 protooncogene in liver cirrhosis and hepatocellular carcinoma. Hepatol Res 2004;29:113-121.

30. Roberts PJ, Der CJ. Targeting the Raf-MEK-ERK mitogen-activated protein kinase cascade for the treatment of cancer. Oncogene 2007;26:3291-310.

31. Tsuboi Y, Ichida T, Sugitani S, et al. Overexpression of extracellular signal-regulated protein kinase and its correlation with proliferation in human hepatocellular carcinoma. Liver Int 2004;24:432-6.

32. Yoshida T, Hisamoto T, Akiba J, et al. Spreds, inhibitors of the Ras/ ERK signal transduction, are dysregulated in human hepatocellular carcinoma and linked to the malignant phenotype of tumors. Oncogene 2006;25:6056-66

33. Babu GJ, Lalli MJ, Sussman MA, et al. Phosphorylation of elk-1 by MEK/ERK pathway is necessary for c-fos gene activation during cardiac myocyte hypertrophy. J Mol Cell Cardiol 2000;32:1447-57.

34. Shaulian E, Karin M. AP-1 in cell proliferation and survival. Oncogene 2001;20:2390-400.

35. Ito $Y$, Sasaki $Y$, Horimoto $M$, et al. Activation of mitogen-activated protein kinases/extracellular signal-regulated kinases in human hepatocellular carcinoma. Hepatology 1998;27:951-8.

36. Mebratu Y, Tesfaigzi Y. How ERK1/2 activation controls cell proliferation and cell death: Is subcellular localization the answer? Cell Cycle 2009;8:1168-75

37. Bonni A, Brunet A, West AE, et al. Cell survival promoted by the RasMAPK signaling pathway by transcription-dependent and -independent mechanisms. Science 1999;286:1358-62.

38. Thiery JP, Acloque H, Huang RY, et al. Epithelial-mesenchymal transitions in development and disease. Cell 2009;139:871-90.

39. Serrano-Gomez SJ, Maziveyi M, Alahari SK. Regulation of epithelialmesenchymal transition through epigenetic and post-translational modifications. Molecular cancer 2016;15:18-18.

40. TripathiK, Garg M. Mechanistic regulation of epithelial-to-mesenchymal transition through RAS signaling pathway and therapeutic implications in human cancer. Journal of cell communication and signaling 2018;12:513527

41. Chakraborti S, Mandal M, Das S, Mandal A, Chakraborti T. Regulation of matrix metalloproteinases: an overview. Mol Cell Biochem 2003;253:26985.

42. Chen H, Zhu G, Li Y, et al. Extracellular signal-regulated kinase signaling pathway regulates breast cancer cell migration by maintaining slug expression. Cancer Res 2009;69:9228-35.

43. Graham TR, Zhau HE, Odero-Marah VA, et al. Insulin-like growth factorI-dependent up-regulation of ZEB1 drives epithelial-to-mesenchymal transition in human prostate cancer cells. Cancer Res 2008;68:2479-88.

44. Shin S, Blenis J. ERK2/Fra1/ZEB pathway induces epithelial-tomesenchymal transition. Cell Cycle. 2010; 9:2483-2484.

45. Smit MA, Peeper DS. Epithelial-mesenchymal transition and senescence: two cancer-related processes are crossing paths. Aging 2010;2:735-741. 
46. Browne G, Sayan AE, Tulchinsky E. ZEB proteins link cell motility with cell cycle control and cell survival in cancer. Cell Cycle 2010;9:886-91.

47. Shin S, Dimitri CA, Yoon SO, et al. ERK2 but not ERK1 induces epithelial-to-mesenchymal transformation via DEF motif-dependent signaling events. Mol Cell 2010;38:114-27.

48. Jungst C, Cheng B, Gehrke R, Schmitz V, Nischalke HD, Ramakers J et al. Oxidative damage is increased in human liver tissue adjacent to hepatocellular carcinoma. Hepatology 2004; 39: 1663-72.

49. Yagoda N, von Rechenberg M, Zaganjor E, Bauer AJ, Yang WS, Fridman DJ et al. RAS-RAF-MEK-dependent oxidative cell death involving voltage-dependent anion channels. Nature 2007; 447: 864-68.

50. Tai P, Ascoli M. Reactive oxygen species (ROS) play a critical role in the cAMP-induced activation of Ras and the phosphorylation of ERK1/2 in Leydig cells. Mol Endocrinol 2011; 25: 885-93.

51. Weinberg F, Hamanaka R, Wheaton WW, Weinberg S, Joseph J, Lopez $\mathrm{M}$ et al. Mitochondrial metabolism and ROS generation are essential for Kras-mediated tumorigenicity. Proc Natl Acad Sci USA 2010; 107: 8788-93.

52. Wang AG, Moon HB, Lee MR, Hwang CY, Kwon KS, Yu SL et al. Gender-dependent hepatic alterations in H-ras $12 \mathrm{~V}$ transgenic mice. J Hepatol 2005; 43: 836-44.

53. Wang AG, Moon HB, Chae JI, Kim JM, Kim YE, Yu DY et al. Steatosis induced by the accumulation of apolipoprotein A-I and elevated ROS levels in H-ras12V transgenic mice contributes to hepatic lesions. Biochem Biophys Res Commun 2011; 409: 532-38.

54. Saha B, Nandi D. Farnesyltransferase inhibitors reduce Ras activation and ameliorate acetaminophen-induced liver injury in mice. Hepatology 2009; 50: $1547-57$.

55. Cullen PJ, Lockyer PJ. Integration of calcium and Ras signaling. Nat Rev Mol Cell Biol 2002; 3:339 -48.
56. Buday L, Downward J. Many faces of Ras activation. Biochim Biophys Acta 2002; 1786:178 -87.

57. Veal EA, Day AM, Morgan BA. Hydrogen peroxide sensing and signaling. Mol Cell 2007; 26:1-14.

58. Myers TJ, Brennaman LH, Stevenson M, Higashiyama S, Russell WE, Lee DC, Sunnarborg SW. Mitochondrial reactive oxygen species mediate GPCR - induced TACE/ADAM17 - dependent transforming growth factor - shedding. Mol Biol Cell 2009; 20:5236-49.

59. Kim EK, Choi EJ. Compromised MAPK signaling in human diseases: an update. Arch Toxicol. 2015; 89:867-82.

60. Barthel A, Klotz LO. Phosphoinositide 3-kinase signaling in the cellular response to oxidative stress. Biol Chem. 2005; 386:207-16.

61. McCubrey JA, Lahair MM, Franklin RA. Reactive oxygen speciesinduced activation of the MAP kinase signaling pathways. Antioxid Redox Signal. 2006; 8:1775-89.

62. Wagner EF, Nebreda AR. Signal integration by JNK and p38 MAPK pathways in cancer development. Nat Rev Cancer. 2009; 9:537-49.

63. Dhillon AS, Hagan S, Rath O, Kolch W. MAP kinase signalling pathways in cancer. Oncogene. 2007; 26:3279-90.

64. Yang Q, Lee JD. Targeting the BMK1 MAP kinase pathway in cancer therapy. Clin Cancer Res. 2011; 17:3527-32.

65. Shahin YR, Elguindy NM, Abdel Bary A, Balbaa M. The protective mechanism of Nigella sativa against diethylnitrosamine-induced hepatocellular carcinoma through its antioxidant effect and EGFR/ ERK1/2 signaling. Environ Toxicol. 2018; 33:885-98.

Citation: Mahmoud Balbaa, Yasmin R. Shahina, Nihal M. Elguindy, "Cell Signaling in Hepatocellular Carcinoma", American Research Journal of Biosciences, vol 6, no. 1, 2020, pp. 1-6.

Copyright (ㄷ) 2020 Mahmoud Balbaa, et al. This is an open access article distributed under the Creative Commons Attribution License, which permits unrestricted use, distribution, and reproduction in any medium, provided the original work is properly cited. 